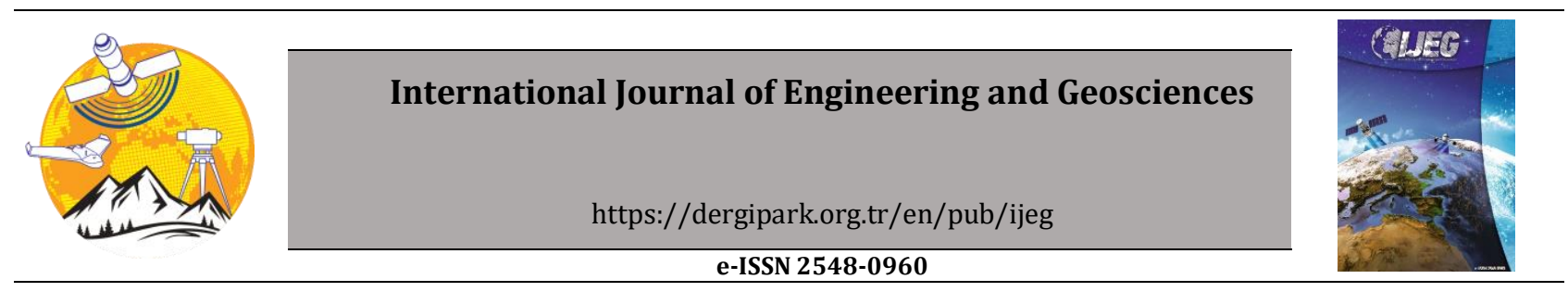

\title{
Accuracy assessment of digital surface models from unmanned aerial vehicles' imagery on archaeological sites
}

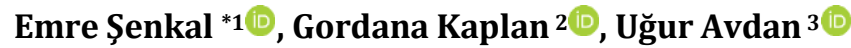 \\ ${ }^{1}$ Eskisehir Technical University, Remote Sensing and Geographical Information Systems Department, Eskisehir, Turkey \\ 2Eskisehir Technical University, Earth and Space Sciences Institute, Eskisehir, Turkey
}

\author{
Keywords \\ Unmanned aerial vehicle \\ Digital surface model \\ Accuracy analysis \\ GIS \\ Remote sensing
}

\begin{abstract}
With the developing technologies, the use of unmanned aerial vehicles's (UAV) is increasing in all areas. Compared with the conventional photogrammetry and remote sensing sensors, UAVs are more convenient to collect data for small areas. In this study, the accuracy of UAV products was investigated in the archeological area of Eskișehir Şarhöyük. In order to produce reference data for the orthophoto and DTM accuracy analysis, a digital map from the test area was produced using in-situ measurements. Also, for the comparison of the point cloud, a small test area was determined and reference point cloud data was collected with terrestrial laser scanner. The comparison of the results showed significant difference between the UAV images and images collected by conventional methods. Thus, while there was $1 \mathrm{~m}$ difference between the data without the use of control points, and the use of control points significantly improved the results.
\end{abstract}

\section{INTRODUCTION}

With the rapid development of remote sensing technologies, Unmanned Aerial Vehicles (UAVs) have been widely used in many different research areas producing high-resolution data, including Digital Surface Models (DSMs) and orthorectified images (orthophotos) (Gindraux et al. 2017). The wide range of application include but are not limited to; agriculture (Costa et al. 2012), ecological studies (Anderson and Gaston 2013), water resource management (DeBell et al. 2016), glacier monitoring (Fugazza et al. 2015), soil erosion (d'OleireOltmanns et al. 2012), landslide mapping (Comert et al. 2019), photogrammetric remote sensing and geoinformation (Colomina and Molina 2014), building extraction (Comert and Kaplan 2018, Comert et al. 2018) etc.

UAV data has also been used for mapping and monitoring archeological areas (Tscharf et al. 2015, Holness et al. 2016, Themistocleous 2017). Thus, here we give brief literature review of the archeological studies conducted with UAV data. (Eisenbeiss and Zhang 2006) compared DSM from UAV and terrestrial laser scanner in the Pinchango Alto archaeological field. The results showed that the height modes were substantially consistent with each other. In their study, (Sauerbier and Eisenbeiss 2010) used two different UAVs for documenting and monitoring excavations in three archaeological sites. The results of the study showed that the data obtained with UAV can be successfully used for documenting archaeological and cultural heritage. (Lin et al. 2011) used satellite imagery, UAV, and ground radar for detection of archaeological anomalies in three different archaeological sites in north Mongolia. The results showed that satellite imagery from Geo-Eye 1 can be used for objects long 1 - $10 \mathrm{~m}$, while for smaller objects, UAV data should be used. Ground radar data can be used in order to obtain additional data about the archaeological remaining underground. Aiming to test UAV use in archeological areas, (Chiabrando et al. 2011) used a small remote controlled helicopter and a small 
aircraft over the Reggia di Venaria Reale and Augusta Bagiennorum sites in Italy. The experiments carried out from 100 and 60 meter heights from the small aircraft, and the 50 and 15 meters' heights flights were used to produce $1 / 200$ and $1 / 100$ scale orthophoto and digital maps, respectfully. As a result of the study, it was revealed that UAVs are useful for producing large scale maps needed in archaeological documentation. In addition, the low cost and speed of data collection has been shown to be suitable for archaeological survey studies. Using a camera placed on a helium balloon, collected data and obtained DTM and 3 Dimensional model of the archeological area of Cerrillo Blanco in Spain. As a result of the study, it was concluded that the balloon system used in the scope of the study is suitable for mapping small and medium sized archaeological sites in areas where the wind effect can be controlled.

In more recent studies, researchers have used UAV technology with conjunction with geoinformation systems (GIS) and ground positioning systems (GPS) for protection and management of cultural heritage and archeological sites (Tache et al. 2018). Similar studies have been conducted for several other sites in Turkey (Ilci et al. 2019), Patara, Jordan (Hasting 2019) etc. Combination of UAV and Ground Penetrating Radar (GPR) has been used in order to detect non-invasive detection of buried objects (Garcia-Fernandez et al. 2018).

However, not many studies can be found evaluating the accuracy of the produced UAV maps. (Rusli et al. 2019) compared accuracy of DEM obtained from UAV and TanDEM-X satellite sensor. The results indicated difference of 3 to 4 in the DEMs. Perez et al. (Pérez et al. 2019) did an investigation concerning the positional accuracy and maximum allowable scale of UAV products for archaeological site documentation.

The main aim of this study is to investigate the accuracy of UAV products (ortophoto image, DSM, point cloud) produced from processed photographs obtained from UAV. The investigation was made over the Şarhoyük archeological site in Eskisehir, Turkey. Thus, data acquisition from different heights and different overlays were performed. The aim of image acquisition at different heights and different overlays is to investigate the effect of height and overlay on the resulting image.

In order to investigate the accuracy of the produced data, ground control points (GCP) were placed in the pre-flight area and the coordinates of these points were determined precisely by the geodetic GNSS receiver. Some of the control points were used to coordinate the produced orthophoto and DSM, while other control points were used in the comparison process for the accuracy analysis of the orthophoto images. In addition, for the accuracy analysis of the DSM obtained from the UAV, the DSM of the study area was produced by topographic method. The numerical surface model created by geodetic method and surface models created by unmanned aerial vehicle were compared.

The main purpose of this study is to investigate the accuracy of the final products produced from images obtained by UAV. For this purpose, the accuracy of the orthophoto, point cloud and DSM to be produced from UAV were compared with data collected with conventional methods.

\section{METHODOLOGY}

\subsection{Study Area}

The ancient city of Dorylaeum or Dorylaion, or Şarhoyük in Turkish, is the oldest settlement in the northeast of Eskişehir. With 17 meters' heights, it is one of the largest mounds in Central Anatolia. About $1 \mathrm{~km}$ west of the lower city, there is a necropolis which was founded around the mound. The excavations yielded finds from the Early Bronze Age, Hittite, Phrygian, Hellenistic, Roman, Byzantine and Ottoman periods. According to William Mitchell Ramsay, after Dorylaion was abandoned, a new settlement was established in the south of the city and the region where Dorylaion was located was called Eskişehir (Old Town). The reason for the selection of Sharhoyuk as a study area in this paper, are the different heights of the land, the number of excavation and filling areas on the land, and the fact that this is a protected archeological site where the human effects are lower than other areas.

\subsection{Data and Methods}

In order to provide the relationship between images obtained from the UAV and the ground, white cross with red dot GCPs were deployed and fixed on the archeological site. The GCP center were fixed prior to the UAV flights with a Javad TRIUMPH geodetic Global Navigation Satellite System (GNSS) receiver. The GCPs were measured before the flight. The GCPs measurement was executed in real-time kinematic (RTK) mode using virtual reference stations from the permanent GNSS station network of Turkey (TUSAGA-Active Turkish National Permanent GPS Active Stations Network). From repeated measurements of fixed locations, it was estimated that the mean accuracy of the measurements is $1-2 \mathrm{~cm}$. In flat areas of the study area, the GCPs measurements were made at approximately 10 meters and less than 10 meters in non-flat areas. Each point was measured in five epochs. As a results, 5965 GCPs were deployed in the study area (Figure 2). excavation and filling areas on the land, and the fact that this is a protected archeological site where the human effects are lower than other areas. The study area is presented in Figure 1. 


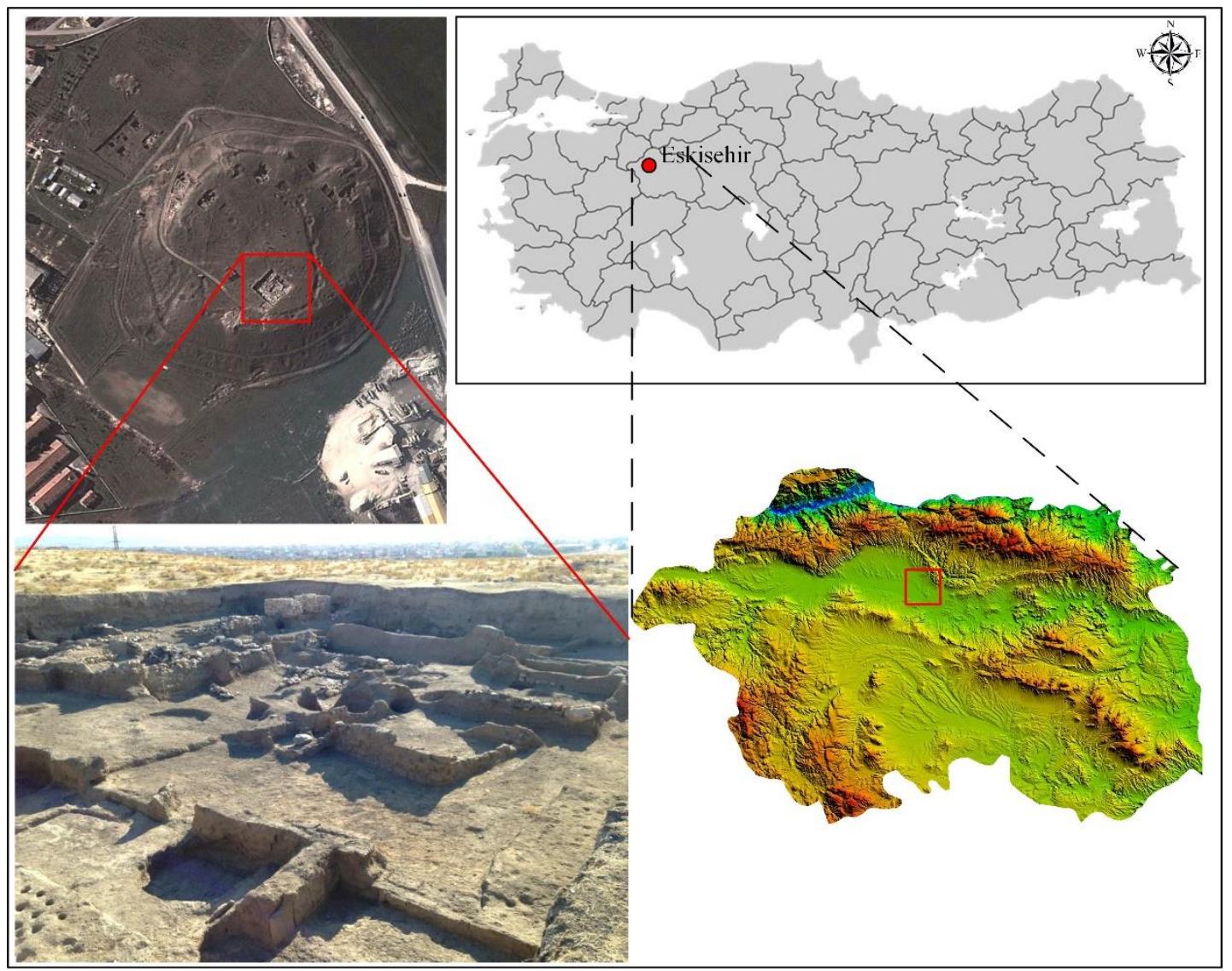

Figure 1. Study area; Şarhoyük archeological site, Eskisehir, Turkey

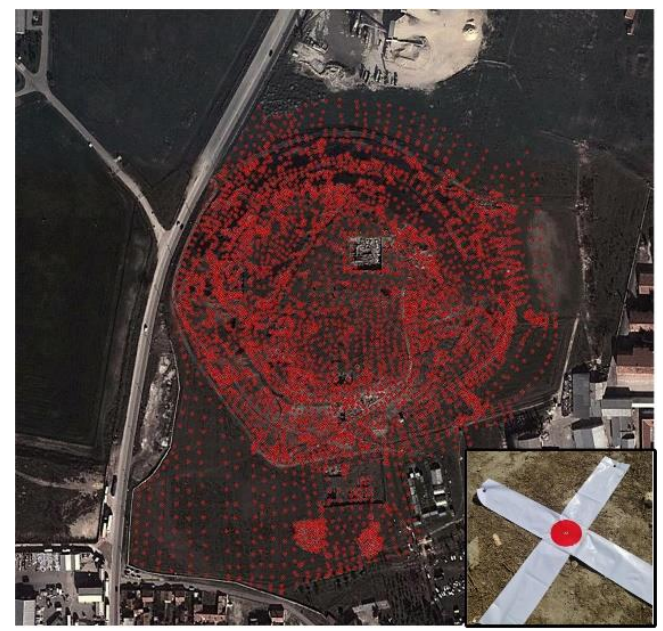

Figure 2. GCPs over the study area

For the image acquisition, SenseFly eBee UAV was used. The flight was automatically carried out and arranged according to the prepared flight plan. Technical specifications of the used UAV are given in Table 1. Two Canon cameras were used during the data acquisition, Canon IXUS 125 HS and Canon PowerShot ELPH 110 HS. The main difference between the two cameras is the spectral range. While the first camera operates in the Red, Green, Blue (RGB) part, the second camera operated in the Near Infrared, Green, Blue (NIRGB) part of electromagnetic spectrum. During the image acquisition, an on-board GPS and an inertial measurement unit provide information about the approximate 3D position, roll, pitch and yaw of the UAV.

Table 1. Technical specification of the UAV used in this study

\begin{tabular}{ll}
\hline Wing Span & $96 \mathrm{~cm}$ \\
Weights & $700 \mathrm{~g}$ \\
Active time & $\sim 45 \mathrm{~min}$ \\
Flight speed & $36-57 \mathrm{~km} / \mathrm{h}$ \\
Radio range & $3 \mathrm{~km}$ \\
Covering area & $1.5-10 \mathrm{~km}^{2}$ \\
Spatial resolution & $3-30 \mathrm{~cm}$ \\
\hline
\end{tabular}

Flights were planned with the software eMotion 2.4 provided by SenseFly. A minimum of $60 \%$ lateral and $70 \%$ longitudinal ground overlap was ensured between adjacent images. The first step in the flight planning was to determine the height of the flight. The UAV used in this study has a capability of flying between 50 and 1000 meters, with a spatial resolution of the images between 2 and $40 \mathrm{~cm}$. After determining the flight height, the flight operation should be prepared taking into consideration the overlap ratios of the image frames, depending on the area covered in the field. The flight preparation was made as recommended in (Eisenbeiß 2009, Karakış 2012).

In order to obtain photogrammetric images of the study area, three different flights were prepared with the e-Motion2 software. Details about each flight are given in Table 2. 
The images obtained from the field were processed with PostFlight Terra 3D software. The data processing process consists of three steps:

i) Initial Processing

ii) ii) Point Cloud Densification

iii) iii) Digital Surface Model and Orthomosaic Production.

In order to investigate the accuracy of the data obtained from the UAV, the data were processed in four different ways. First, the data were processed without a GCPs, then the study area was divided into three levels: low level, medium level and high level depending on the terrain height (Figure 3). The purpose of this process is to observe the effects of GCPs over the results within a certain height range. Using the GCPs located at these three levels, three different data manipulations were performed for the results of each flight. Afterwards, data were processed by using low, medium and high level GCPs from the existing control points (Figure 3).

Table 2. Flight details

\begin{tabular}{llll}
\hline Parameter & 1. Flight & 2. Flight & 3. Flight \\
\hline Camera & RGB & NIRGB & RGB \\
Terrain mode & Easy & Easy & Difficult \\
Flight height & $130 \mathrm{~m}$ & $130 \mathrm{~m}$ & $196 \mathrm{~m}$ \\
Ground Sample Range & $4 \mathrm{~cm}$ & $4 \mathrm{~cm}$ & $6 \mathrm{~cm}$ \\
Lateral Overlap & $60 \%$ & $60 \%$ & $85 \%$ \\
Longitudinal Overlap & $70 \%$ & $70 \%$ & $70 \%$ \\
Image Number & 105 & 101 & 137 \\
Flight time & $13 \mathrm{~min}$ & $12 \mathrm{~min}$ & $22 \mathrm{~min}$ \\
\hline
\end{tabular}
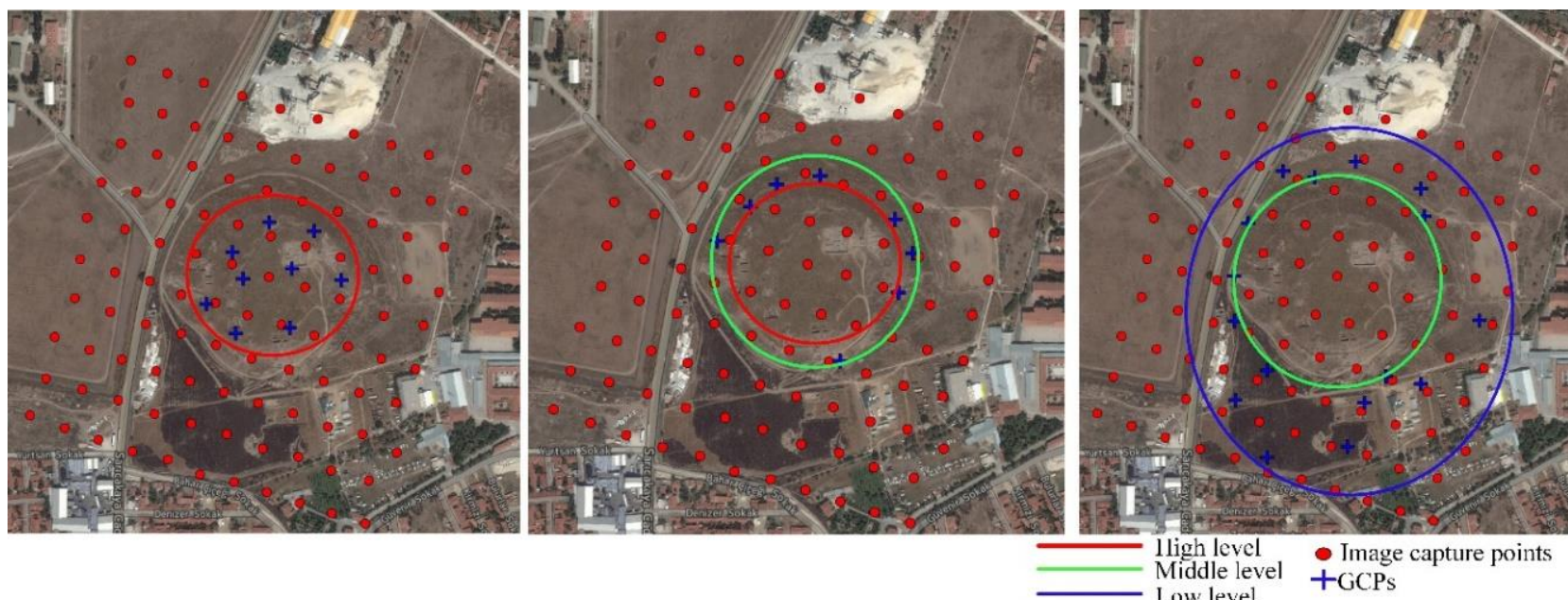

Figure 3. GCPs on different levels on the study area

\section{RESULTS and DISCUSSION}

The coordinates obtained from the field measurements with the GNSS receiver were compared with the coordinates of the digitized GCPs over the orthophoto image obtained from the UAV. First, the coordinates of the GCPs over the product produced without GCPs were compared with the coordinates measured at site. The differences from the 34 GCPs, from both easy and difficult flight mode, used on the three different levels (Figure 3), are shown in Table 3.

The same analyses were conducted for all six projects between the measured GCPs and the coordinated from the product obtained with the use of GCPs. The differences between the coordinates of the measured GCPs and the GCPs from the three different levels (low, medium, high) were compared with the results from both easy and difficult flight results. For this purpose, the GCPs from specific level were excluded and the GCPs from the two other levels were used for the evaluation of the results. The results are presented in Table 4 and Table 5 .

From the comparison of the results, it has been seen that the GCPs in the middle and high levels have more difference in comparison with the GCPs in the low level of the study area. In comparison of the two different terrain models, there was no significant difference noticed between the results.

\subsection{Field Data Comparison}

Four of the eleven excavation sites in the study area were selected for the comparison of the field data (Figure $4-a$ ). In order to minimize the error, each edge of the selected excavation was measured using the same points on the raster. As a result of the measurements, no significant difference was found between the terrain data and the data obtained from the UAV and the produced raster images. The use of 
GCPs or flight height difference did not have a significant effect on length in the selected area. In the point-to-point comparison process, approximately 1 meter offset was detected between the raster data produced with and without GCPs (Figure $4-\mathrm{b}$ ). This offset does not affect the length as it acts in the same direction on the edges of the excavation area.

In order to compare the DSM results and to determine the product with least error, different GCPs have been compared. In addition, in order to investigate the effect of the flight height in the DSM production, comparison of the results obtained from flights with different height has been made. The results show the height differences for pixel based DSM. By examining in the areas of the height differences overlap, it is possible to determine the accuracy of the numerical surface model. In order to compare the produced products with field measurements, a DSM was produced from the 5965 points field measured with GNSS receiver. The results are presented in Figure 5. As stated before, the DSM from the UAV data collection was compared with the field measurements. The two different DSM models were compared in order to investigate the effect of the obtained results. The result of the comparison indicates that there is high overlap in the areas obtained with GCPs. High differences are being notices over the areas where there is low overlap between the images, especially on the edges of the study area. The difference between the DSM created with all GCPs with easy and difficult terrain mode are presented in Figure 6.

Table 3. Difference between the GCPs

\begin{tabular}{|c|c|c|c|c|c|c|}
\hline \multirow{2}{*}{ No } & \multicolumn{3}{|c|}{ Easy terrain mode } & \multicolumn{3}{|c|}{ Difficult flight mode } \\
\hline & $\Delta \mathrm{Y}$ & $\Delta \mathrm{X}$ & $\Delta \mathrm{Z}$ & $\Delta \mathrm{Y}$ & $\Delta \mathrm{X}$ & $\Delta \mathrm{Z}$ \\
\hline GCP1 & -0.308 & -0.703 & 0.860 & 0.795 & 0.125 & 0.498 \\
\hline GCP2 & -0.127 & -0.742 & 0.751 & 1.172 & -0.182 & 0.539 \\
\hline GCP3 & -0.084 & -0.766 & 0.789 & 1.333 & -0.258 & 0.549 \\
\hline GCP4 & 0.020 & -0.833 & 0.806 & 1.567 & -0.416 & 0.662 \\
\hline GCP5 & 0.048 & -0.752 & 0.701 & 1.736 & -0.216 & 0.800 \\
\hline GCP6 & 0.002 & -0.617 & 0.622 & 1.749 & 0.078 & 0.880 \\
\hline GCP7 & -0.123 & -0.547 & 0.558 & 1.667 & 0.350 & 0.878 \\
\hline GCP8 & -0.231 & -0.500 & 0.486 & 1.635 & 0.624 & 0.844 \\
\hline GCP9 & -0.145 & -0.476 & 0.443 & 1.879 & 0.612 & 0.912 \\
\hline GCP10 & 0.050 & -0.607 & 0.541 & 2.050 & 0.135 & 0.945 \\
\hline GCP11 & 0.151 & -0.804 & 0.784 & 1.972 & -0.412 & 0.849 \\
\hline GCP12 & 0.101 & -0.937 & 0.856 & 1.636 & -0.701 & 0.530 \\
\hline GCP13 & -0.016 & -0.982 & 0.956 & 1.243 & -0.799 & 0.471 \\
\hline GCP14 & -0.169 & -0.976 & 0.907 & 0.846 & -0.797 & 0.248 \\
\hline GCP15 & -0.347 & -0.936 & 0.973 & 0.456 & -0.577 & 0.186 \\
\hline GCP16 & -0.376 & -0.988 & 1.071 & 0.257 & -0.797 & 0.306 \\
\hline GCP17 & -0.550 & -0.946 & 1.230 & -0.185 & -0.580 & 0.355 \\
\hline GCP18 & -0.639 & -0.770 & 1.262 & -0.078 & 0.062 & 0.272 \\
\hline GCP19 & -0.597 & -0.701 & 1.079 & 0.220 & 0.229 & 0.172 \\
\hline GCP20 & -0.563 & -0.634 & 1.019 & 0.439 & 0.380 & 0.274 \\
\hline GCP21 & -0.632 & -0.592 & 1.099 & 0.371 & 0.627 & 0.380 \\
\hline GCP22 & -0.479 & -0.748 & 0.948 & 0.503 & 0.136 & 0.302 \\
\hline GCP23 & -0.184 & -0.626 & 0.678 & 1.185 & 0.151 & 0.620 \\
\hline GCP24 & -0.054 & -0.649 & 0.664 & 1.496 & 0.015 & 0.821 \\
\hline GCP25 & -0.142 & -0.603 & 0.595 & 1.447 & 0.262 & 0.825 \\
\hline GCP26 & -0.267 & -0.522 & 0.695 & 1.152 & 0.475 & 0.708 \\
\hline GCP27 & -0.433 & -0.562 & 0.766 & 0.931 & 0.574 & 0.629 \\
\hline GCP28 & -0.586 & -0.412 & 0.834 & 0.870 & 1.095 & 0.710 \\
\hline GCP29 & -0.332 & -0.516 & 0.641 & 1.255 & 0.706 & 0.729 \\
\hline GCP30 & -0.127 & -0.477 & 0.542 & 1.595 & 0.626 & 0.828 \\
\hline GCP31 & 0.082 & -0.745 & 0.661 & 1.899 & -0.202 & 0.839 \\
\hline GCP32 & 0.024 & -0.888 & 0.886 & 1.366 & -0.640 & 0.568 \\
\hline GCP33 & -0.158 & -0.891 & 0.830 & 0.959 & -0.484 & 0.309 \\
\hline GCP34 & -0.258 & -0.824 & 0.854 & 0.761 & -0.268 & 0.383 \\
\hline $\begin{array}{c}\text { Square Mean } \\
\text { Error }\end{array}$ & 0.318 & 0.733 & 0.830 & 1.272 & 0.503 & 0.630 \\
\hline
\end{tabular}


Table 4. Difference between measured and GCPs obtained from easy terrain mode

\begin{tabular}{|c|c|c|c|c|c|c|c|c|c|}
\hline \multirow{3}{*}{ No } & \multicolumn{9}{|c|}{ Easy terrain mode } \\
\hline & \multicolumn{3}{|c|}{ Low terrain } & \multicolumn{3}{|c|}{ Middle terrain } & \multicolumn{3}{|c|}{ High terrain } \\
\hline & $\Delta \mathrm{Y}$ & $\Delta \mathrm{X}$ & $\Delta \mathrm{Z}$ & $\Delta \mathrm{Y}$ & $\Delta \mathrm{X}$ & $\Delta \mathrm{Z}$ & $\Delta \mathrm{Y}$ & $\Delta \mathrm{X}$ & $\Delta \mathrm{Z}$ \\
\hline GCP1 & 0.028 & -0.025 & 0.029 & 0.018 & 0.030 & 0.000 & GCP & GCP & GCP \\
\hline GCP2 & 0.033 & 0.006 & -0.044 & 0.019 & 0.040 & -0.072 & GCP & GCP & GCP \\
\hline GCP3 & 0.010 & 0.005 & 0.076 & -0.014 & 0.029 & 0.018 & GCP & GCP & GCP \\
\hline GCP4 & -0.021 & -0.040 & 0.042 & GCP & GCP & GCP & 0.028 & -0.066 & 0.087 \\
\hline GCP5 & 0.027 & -0.008 & -0.009 & GCP & GCP & GCP & 0.053 & -0.046 & 0.024 \\
\hline GCP6 & 0.032 & 0.014 & -0.013 & GCP & GCP & GCP & 0.036 & -0.012 & -0.005 \\
\hline GCP7 & 0.019 & 0.015 & 0.057 & GCP & GCP & GCP & -0.035 & 0.003 & -0.029 \\
\hline GCP8 & $\mathrm{GCP}$ & $\mathrm{GCP}$ & GCP & -0.068 & -0.049 & -0.093 & -0.074 & -0.055 & -0.099 \\
\hline GCP9 & GCP & GCP & GCP & -0.082 & -0.058 & -0.091 & -0.078 & -0.046 & -0.105 \\
\hline GCP10 & GCP & GCP & GCP & -0.042 & -0.052 & -0.072 & 0.017 & -0.037 & -0.028 \\
\hline GCP11 & GCP & GCP & GCP & -0.019 & -0.069 & -0.030 & 0.060 & -0.068 & 0.096 \\
\hline GCP12 & GCP & GCP & GCP & -0.004 & -0.058 & -0.020 & 0.071 & -0.075 & 0.102 \\
\hline GCP13 & GCP & GCP & GCP & 0.003 & 0.021 & -0.060 & 0.054 & -0.035 & 0.070 \\
\hline GCP14 & GCP & GCP & GCP & -0.011 & 0.090 & -0.214 & 0.026 & 0.020 & -0.047 \\
\hline GCP15 & GCP & GCP & GCP & 0.003 & 0.120 & -0.198 & 0.004 & 0.041 & -0.084 \\
\hline GCP16 & GCP & GCP & GCP & 0.014 & 0.200 & -0.351 & 0.014 & 0.112 & -0.194 \\
\hline GCP17 & GCP & GCP & GCP & 0.066 & 0.256 & -0.384 & 0.040 & 0.148 & -0.267 \\
\hline GCP18 & GCP & GCP & GCP & 0.053 & 0.178 & -0.105 & 0.006 & 0.110 & -0.054 \\
\hline GCP19 & GCP & GCP & GCP & 0.003 & 0.109 & -0.027 & -0.047 & 0.086 & 0.005 \\
\hline GCP20 & GCP & GCP & GCP & -0.010 & 0.104 & 0.031 & -0.068 & 0.053 & 0.072 \\
\hline GCP21 & GCP & GCP & GCP & -0.005 & 0.048 & 0.082 & -0.068 & 0.014 & 0.111 \\
\hline GCP22 & 0.017 & -0.049 & -0.007 & GCP & GCP & GCP & -0.040 & -0.004 & 0.009 \\
\hline GCP23 & 0.040 & 0.002 & -0.047 & -0.007 & 0.025 & -0.020 & GCP & GCP & GCP \\
\hline GCP24 & -0.016 & 0.013 & -0.086 & -0.030 & 0.009 & -0.055 & GCP & GCP & GCP \\
\hline GCP25 & 0.035 & -0.035 & -0.070 & -0.025 & -0.026 & -0.078 & GCP & GCP & GCP \\
\hline GCP26 & 0.046 & 0.012 & -0.013 & 0.044 & 0.043 & -0.024 & GCP & GCP & GCP \\
\hline GCP27 & -0.033 & -0.037 & 0.041 & GCP & GCP & GCP & -0.078 & -0.018 & 0.022 \\
\hline GCP28 & GCP & GCP & GCP & -0.072 & -0.006 & 0.011 & -0.131 & -0.018 & 0.038 \\
\hline GCP29 & 0.000 & -0.056 & 0.015 & GCP & GCP & GCP & -0.072 & -0.064 & -0.033 \\
\hline GCP30 & 0.109 & 0.041 & -0.052 & GCP & GCP & GCP & 0.035 & 0.005 & -0.086 \\
\hline GCP31 & GCP & GCP & GCP & -0.010 & -0.069 & -0.066 & 0.038 & -0.067 & 0.024 \\
\hline GCP32 & 0.039 & -0.003 & 0.001 & GCP & GCP & GCP & 0.060 & -0.021 & 0.064 \\
\hline GCP33 & 0.013 & -0.056 & -0.017 & 0.004 & 0.023 & -0.131 & GCP & GCP & GCP \\
\hline GCP34 & 0.002 & -0.043 & -0.020 & -0.012 & 0.029 & -0.123 & GCP & GCP & GCP \\
\hline SME & 0.037 & 0.029 & 0.043 & 0.035 & 0.093 & 0.135 & 0.057 & 0.061 & 0.091 \\
\hline
\end{tabular}
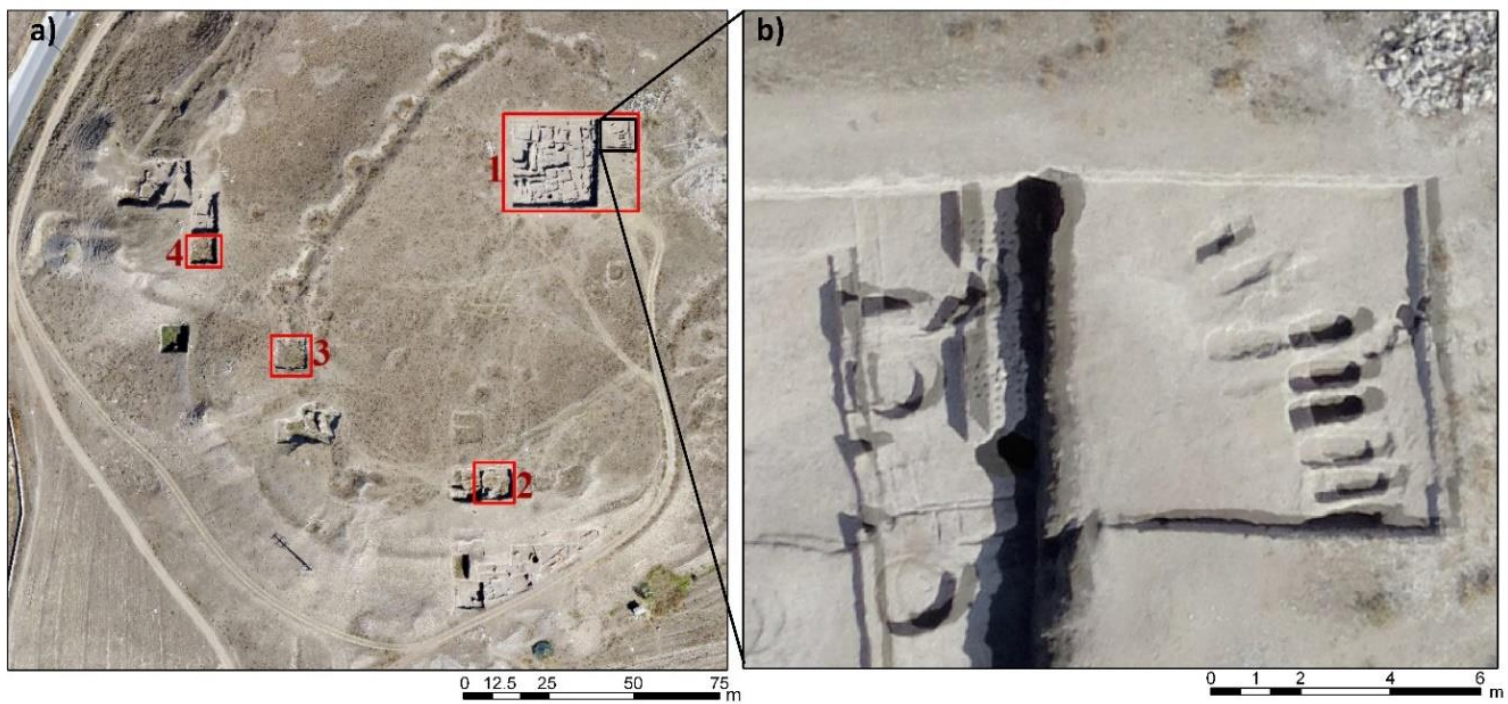

Figure 4. Field data comparison; a) Compared excavation sites; b) Difference between dataset with and without GCPs 

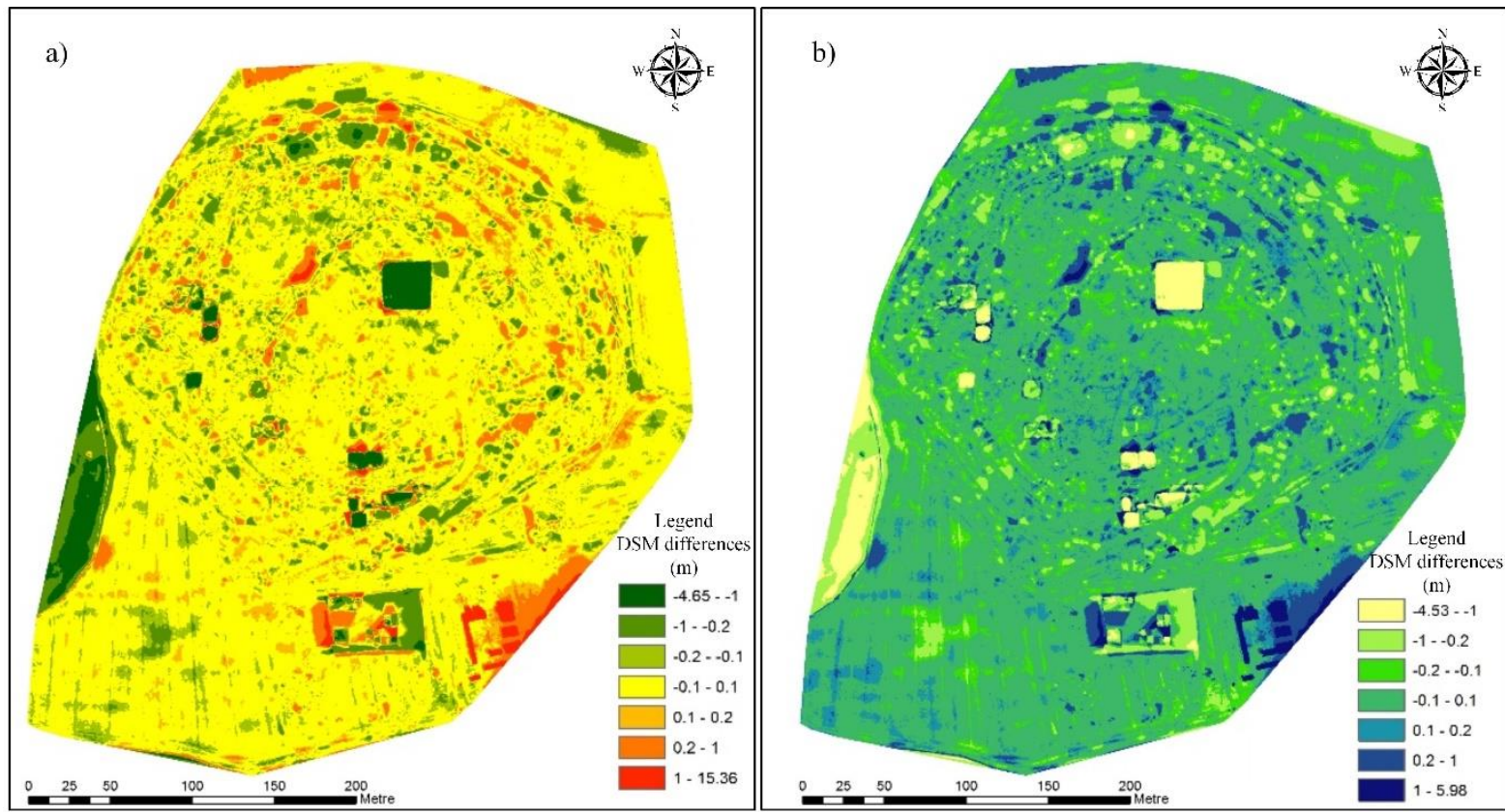

Figure 5. Difference between DSM and field measurements; a) Difficult terrain mode; b) Easy terrain mode

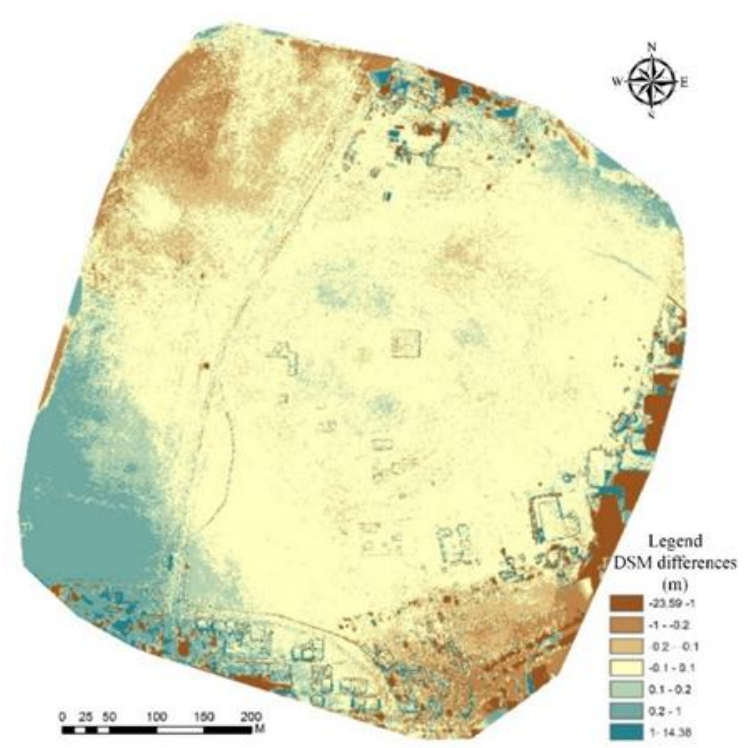

Figure 6. Difference between DSMs created with all GCPs using easy and difficult terrain mode.

The overall results showed that the lowest error of the UAV products is obtained with the use of the low level GCPs. The main reason is that low level GCP are better spread over the study area. The control points at the middle and high levels appear to be clustered. When the square mean error of the products produced without the GCPs is examined, it can be seen that the least error rate is obtained with the flight in difficult terrain mode. The main reason for this is that, in difficult terrain mode, the volume of data is higher and more data is obtained from the field.

From the DSM comparison it can be concluded that the UAV results are in agreement with the field measurements. Thus, there is no significant difference (higher than $10 \mathrm{~cm}$ ) between the results. High differences can be noticed in areas without measurements. In order to avoid damaging the excavation areas, no data was collected from these parts.

\section{CONCLUSION}

UAVs have been widely used in many different research areas producing high-resolution data, including DSMs and orthorectified images. The evaluation of the accuracy of the UAV maps hasn't been addressed in many studies. Thus, the main objective in this study was the investigation of the accuracy of UAV products. For that purpose, UAV data from different heights and different overlays were collected over an archeological site in Eskisehir, Turkey. In order to investigate the accuracy of the produced data, 5965 GCP were placed in the study area.

The finding of the study indicates that the difference between the UAV data and the field measurements is different with and without GCPs. Thus, while there was approximately $1 \mathrm{~m}$ difference between the coordinates obtained by terrestrial method and UAV, with the use of GCPs this difference has been lowered to $5 \mathrm{~cm}$. One of the most important features of UAVs is the quick, precise and in a low cost of data collection without damaging the archaeological area.

As a result of length and area comparisons, UAVs were found to be as reliable as terrestrial measurements. In fact, they provide great advantages to the users by avoiding the human error factor that may occur during terrestrial measurement. The comparison of the DSMs, showed that the differences between terrestrial measurements and UAV measurements are generally $\pm 10 \mathrm{~cm}$ in the $\mathrm{Z}$ axis. It was determined that the height of the GCPs used in the comparison of DSM did not have much effect on the final product. Contrary to the height, the number of GCPs and the 
distribution pattern were found to be more important.

\section{ACKNOWLEDMENT}

This research was funded by Anadolu University Scientific Research Projects Commission under the grant no: 1502E084 and it is a part of Emre Senkal's master thesis.

\section{REFERENCES}

Anderson K \& Gaston K J (2013). Lightweight unmanned aerial vehicles will revolutionize spatial ecology. Frontiers in Ecology and the Environment, 11(3), 138-146. DOI:10.1890/120150

Chiabrando F, Nex F, Piatti D \& Rinaudo F (2011). UAV and RPV systems for photogrammetric surveys in archaelogical areas: two tests in the Piedmont region (Italy). Journal of Archaeological Science, 38(3), 697-710. DOI: 10.1016/j.jas.2010.10.022

Colomina I \& Molina P (2014). Unmanned aerial systems for photogrammetry and remote sensing: A review. ISPRS Journal of Photogrammetry and Remote Sensing, 92, 7997. DOI: $10.1016 /$ j.isprsjprs.2014.02.013

Comert R, Avdan U, Gorum T \& Nefeslioglu H A (2019). Mapping of shallow landslides with object-based image analysis from unmanned aerial vehicle data. Engineering Geology 260: 105264. DOI:10.1016/j.enggeo.2019.105264

Comert R \& Kaplan 0 (2018). "Object based building extraction and building period estimation from unmanned aerial vehicle data. ISPRS Annals of the Photogrammetry, Remote Sensing and Spatial Information Sciences 4(3).

Comert R, Matcı D K \& Avdan U (2018). Detection of collapsed building from unmanned aerial vehicle data with object based image classification. Eskișehir Teknik Üniversitesi Bilim ve Teknoloji Dergisi-B Teorik Bilimler 6, 109-116.

Costa F G, Ueyama J, Braun T, Pessin G, Osório F S \& Vargas P A (2012). The use of unmanned aerial vehicles and wireless sensor network in agricultural applications. IEEE International Geoscience and Remote Sensing Symposium, 5045-5048.

d'Oleire-Oltmanns S, Marzolff I, Peter K D \& Ries J B (2012). Unmanned aerial vehicle (UAV) for monitoring soil erosion in Morocco. Remote Sensing 4(11): 3390-3416. DOI: $10.3390 / \mathrm{rs} 4113390$

DeBell L, Anderson K, Brazier R E, King N \& Jones L (2016). Water resource management at catchment scales using lightweight UAVs: Current capabilities and future perspectives. Journal of Unmanned Vehicle Systems 4(1): 730. DOI:10.1139/juvs-2015-0026
Eisenbeiß H (2009). UAV photogrammetry. PHD Thesis, ETH Zurich.

Eisenbeiss H \& Zhang L (2006). Comparison of DSMs generated from mini UAV imagery and terrestrial laser scanner in a cultural heritage application. International Archives of Photogrammetry, Remote Sensing and Spatial Information Sciences 36(5): 90-96.

Fugazza D, Senese A, Azzoni R S, Smiraglia C, Cernuschi M, Severi D \& Diolaiuti G A (2015). High-resolution mapping of glacier surface features. The UAV survey of the Forni Glacier (Stelvio National Park, Italy). Geografia Fisica e Dinamica Quaternaria, 38, 25-33. DOI 10.4461/GFDQ.2015.38.03

Garcia-Fernandez M, Alvarez-Lopez Y, GonzalezValdes B, Rodriguez-Vaqueiro Y, ArboleyaArboleya A, Heras F L \& Pino A (2018). GPR system onboard a UAV for non-invasive detection of buried objects. IEEE International Symposium on Antennas and Propagation \& USNC/URSI National Radio Science Meeting, 1967-1968.

Gindraux S, Boesch R \& Farinotti D (2017). Accuracy assessment of digital surface models from unmanned aerial vehicles' imagery on glaciers. Remote Sensing, 9(2): 186. 10.3390/rs9020186

Hasting L (2019). "Using Unmanned Aerial Vehicle (UAV) Technology for Archaeology: A Case Study of Petra, Jordan." Scholars Week, 30.

Holness C, Matthews T, Satchell K \& Swindell E C (2016). Remote sensing archeological sites through unmanned aerial vehicle (UAV) imaging. IEEE International Geoscience and Remote Sensing Symposium (IGARSS), 66956698. DOI: 10.1109/IGARSS.2016.7730748

Ilci V, Ozulu I M, Bilgi S \& Alkan R M (2019). The usage of unmanned aerial vehicles (UAVs) for 3D mapping of archaeological sites. FEBFresenius Environmental Bulletin, 28(2), 968974.

Karakış S (2012). Searching The Posibilities of Large Scale Photogrammetric Map Production via Model Aircraft. Harita Dergisi, 147, 13-20. (in Turkish)

Lin A Y-M, Novo A, Har-Noy S, Ricklin N D \& Stamatiou K (2011). Combining GeoEye-1 satellite remote sensing, UAV aerial imaging, and geophysical surveys in anomaly detection applied to archaeology. IEEE Journal of selected topics in applied earth observations and remote sensing, 4(4), 870-876.

Pérez J A, Gonçalves G R \& Charro M C (2019). On the positional accuracy and maximum allowable scale of UAV-derived photogrammetric products for archaeological site documentation. Geocarto International, 34(6): 575-585. DOI: 10.1080/10106049.2017.1421714

Rusli N, Majid M R, Razali N F A A \& Yaacob N F F (2019). Accuracy assessment of DEM from UAV and TanDEM-X imagery. IEEE 15th International Colloquium on Signal Processing 
\& Its Applications (CSPA), 127-131, Penang, Malaysia.

Sauerbier M \& Eisenbeiss H (2010). UAVs for the documentation of archaeological excavations. International Archives of Photogrammetry, Remote Sensing and Spatial Information Sciences, 38(5), 526-531.

Tache A V, Sandu I C A, POPESCU O-C \& PETRIŞOR AI (2018). UAV solutions for the protection and management of cultural heritage. Case study: Halmyris Archaeological site. International Journal of Conservation Science, 9(4), 795-804.

Themistocleous K (2017). The use of UAVs to monitor archeological sites: the case study of
Choirokoitia within the PROTHEGO project. Fifth International Conference on Remote Sensing and Geoinformation of the Environment (RSCy2017), International Society for Optics and Photonics. DOI: $10.1117 / 12.2292351$

Tscharf A, Rumpler M, Fraundorfer F, Mayer G \& Bischof $H$ (2015). On the use of UAVs in mining and archaeology-geo-accurate 3D reconstructions using various platforms and terrestrial views. ISPRS Annals of Photogrammetry, Remote Sensing \& Spatial Information Sciences, 2, 15-22.

(c) Author(s) 2021.

This work is distributed under https://creativecommons.org/licenses/by-sa/4.0/ 